\title{
Combined pulmonary fibrosis and emphysema in patients exposed to agrochemical compounds
}

\section{To the Editors:}

In the very interesting article concerning the clinical syndrome resulting from combined pulmonary fibrosis and emphysema (CPFE) published in the European Respiratory Journal, COTTIN et al. [1] speculated that both emphysema and fibrosis might be related to a common environmental trigger and/or genetic factor, with tobacco exposure playing a central role.

Here, we report our clinical experience of the same syndrome in order to emphasise the possible role of environmental exposure in the pathogenesis of the disease. Over the last 2 yrs, 41 patients with pulmonary fibrosis were investigated in our teaching hospital in Greece, which is located in the middle of a large rural area. In total, 21 cases fulfilled the criteria for the diagnosis of idiopathic pulmonary fibrosis, nine patients had an associated connective tissue disease, two had primary biliary cirrhosis, and nine patients met the imaging criteria for CPFE as described previously by COTTIN et al. [1]. All CPFE patients were male and current or ex-smokers. The most interesting point is that all patients were farmers and had significant exposure to agrochemical compounds. It is also important to point out that paraseptal emphysema in the upper lobes was also present in five cases and most prominent in three. It is well known that paraseptal emphysema is associated with tobacco smoking and results in fibrosis-like thickening of the interlobular septa [2]. However, the fact that our patients had the same imaging findings as those described by COTTIN et al. [1] shows that paraseptal emphysema is truly a novel finding and, as these authors suggest, it may be the hallmark of CPFE. Our findings are in complete agreement with those of COTTIN et al. [1]. Recently, CHURG et al. [3] have also described an airway-centred interstitial fibrosis in 12 patients. One of them was a gardener and former smoker exposed to agrochemical compounds. According to the aforementioned observations, it seems possible that exposure to agrochemical compounds could be a potential triggering event, which is capable of causing airway and interstitial disease in probable genetically susceptible smokers.

Finally, we believe that combined pulmonary fibrosis and emphysema is a new characteristic entity that may occur in tobacco-smoking individuals exposed to an environmental trigger.

\section{Z. Daniil, A. Koutsokera and K. Gourgoulianis}

Medical School, University of Thessaly, Larissa, Greece.

\section{REFERENCES}

1 Cottin V, Nunes H, Brillet P-Y, et al. Combined pulmonary fibrosis and emphysema: a distinct underrecognised entity. Eur Respir J 2005; 26: 586-593.

2 Vehmas T, Kivisaari L, Huuskonen MS, Jaakkola MS. Effects of tobacco smoking on findings in chest computed tomography among asbestos-exposed workers. Eur Respir J 2003; 21: 866-871.

3 Churg A, Myers J, Suarez T, et al. Airway-centered interstitial fibrosis: a distinct form of aggressive diffuse lung disease. Am J Surg Pathol 2004; 28: 62-68.

DOI: 10.1183/09031936.06.00124505

\section{A theory explaining time trends in asthma prevalence}

\section{To the Editors:}

I would like to comment on the interesting article by VAN SCHAYCK and SMIT [1], which documents time trends in the prevalence of childhood asthma in a Dutch general practice registry and corresponding data from repeated cross-sectional surveys in another venue within the same country. While they and others have described an apparent levelling off and/or decline in asthma prevalence, a recent report from Denmark describes an increasing prevalence, particularly for nonatopic asthma in young females and adolescents [2]. No satisfactory explanation for these time trends has been offered. I would like to propose a theory that: 1) explains these geographical and temporal variations in asthma; 2) accounts for genetic susceptibility; and 3) offers the real possibility of prevention, improved treatment and even a potential "cure" for asthma and other related chronic obstructive lung diseases.

STRACHAN [3] has pointed out that time trends in asthma incidence and prevalence can only be accounted for by extremely strong environmental factor(s), among which infectious disease pandemics must be acknowledged. For 
example, country-wide epidemics and the increasing seroprevalence of Chlamydia pneumoniae infection in Finland [4] have been accompanied by corresponding country-wide increased asthma medication use in both males and females and all age groups [5]. The growing body of clinical, epidemiological and therapeutic evidence implicating acute, reactivated and chronic infection by C. pneumoniae in asthma $[6,7]$ forms the basis for proposing the theory that a now waning, geographically heterogeneous worldwide $C$. pneumoniae pandemic is responsible for the recent unexplained observations.

According to this theory, genetic susceptibility involves polymorphisms influencing innate/adaptive immunity and other critical functions (e.g. attachment receptors, etc.) that affect host response to $C$. pneumoniae infection to promote asthma and lung remodelling. As an example, in a case-control study, NAGY et al. [8] reported a strong and significant interaction between $C$. pneumoniae immunoglobulin A antibody, a putative marker for chronic infection, and mannosebinding lectin (MBL) allele polymorphisms producing MBL deficiency in children with asthma. C. pneumoniae has been detected in more than half of bronchoalveolar lavage fluids of another group of asthmatic children [9], confirming that the organism is actually present. MBL deficiencies affect up to one quarter of human populations and, perhaps not coincidentally, $20-25 \%$ is the apparent "upper limit" of wheezing prevalence in epidemiological studies.

Acute primary C. pneumoniae infections have been documented to trigger new-onset asthma, which can be successfully rendered asymptomatic after antibiotic treatment [10], raising the possibility of early detection and prevention. Asthma is a strong risk factor for developing chronic obstructive pulmonary disease (COPD) [11] and chronic C. pneumoniae infection is also implicated in COPD [12], further suggesting the possibility of prevention of COPD by early recognition and treatment.

The Chlamydia-asthma theory is receiving growing attention among a segment of the public that is acutely aware of current limitations in asthma treatment (www.asthmastory.com). I suggest that now is the time for asthma researchers to promote the widespread critical evaluation that the Chlamydia-asthma theory deserves.

\section{D.L. Hahn}

Dean Medical Center, Madison, WI, USA.

\section{REFERENCES}

1 van Schayck CP, Smit HA. The prevalence of asthma in children: a reversing trend. Eur Respir J 2005; 26: 647-650.

2 Thomsen SF, Ulrik CS, Larsen K, Backer V. Change in prevalence of asthma in Danish children and adolescents. Ann Allergy Asthma Immunol 2004; 92: 506-511.

3 Strachan DP. Time trends in asthma and allergy: ten questions, fewer answers. Clin Exp Allergy 1995; 25: 791-794.

4 Puolakkainen M, Ukkonen P, Saikku P. The seroepidemiology of Chlamydiae in Finland over the period 1971 to 1987. Epidemiol Infect 1989; 102: 287-295.

5 Klaukka T, Peura S, Martikainen J. Why has the utilization of antiasthmatics increased in Finland? J Clin Epidemiol 1991; 44: 859-863.
6 Hahn DL. Chlamydia pneumoniae, asthma and COPD: what is the evidence? Ann Allergy Asthma Immunol 1999; 83: 271-292.

7 Hahn DL. Role of Chlamydia pneumoniae as an inducer of asthma. In: Friedman H, Yamamoto Y, Bendinelli M, eds. Chlamydia pneumoniae Infection and Disease. New York, Kluwer Academic/Plenum Publishers, 2004; pp. 239-262.

8 Nagy A, Kozma GT, Keszei M, et al. The development of asthma in children infected with Chlamydia pneumoniae is dependent on the modifying effect of mannose-binding lectin. J Allergy Clin Immunol 2003; 112: 729-734.

9 Webley WC, Salva PS, Andrzejewski C, et al. The bronchial lavage of pediatric patients with asthma contains infectious Chlamydia. Am J Respir Crit Care Med 2005; 171: 1083-1088.

10 Hahn DL. Chlamydia/Mycoplasma: do they cause new-onset asthma in adults? In: Johnston SL, Papadopoulos NG, eds. Respiratory Infections in Allergy and Asthma. New York, Marcel Dekker, Inc., 2003; pp. 645-662.

11 Silva GE, Sherrill DL, Guerra S, et al. Asthma as a risk factor for COPD in a longitudinal study. Chest 2004; 126: 59-65.

12 Hahn DL. Chlamydia pneumoniae and the "Dutch Hypothesis". Chest 2002; 122: 1510-1512.

DOI: 10.1183/09031936.06.00119105

From the authors:

We would like to thank D.L. Hahn for his interesting comments on our recent paper in the European Respiratory Journal. No conclusive explanations have been given thus far on the apparent levelling off of asthma prevalence in children. It has been suggested that the underlying cause of the asthma increase in past decades was due to changes towards a westernised lifestyle. However, it is unlikely that the recent observed plateau, or even decrease, would be due to a stabilisation in a westernised lifestyle. The most likely explanation to us seems that a prevalence plateau of all genetically predisposed children has been reached. This means that children who have a genetic predisposition become asthmatic due to relevant exposure. This relevant exposure could indeed be acute primary infections. Moreover, asthmatic children may be diagnosed earlier because of earlier symptom presentation followed by correct diagnosis and therapy. It has previously been shown that underpresentation of asthma symptoms will normally lead to underdiagnosis of asthma, resulting in underestimated asthma prevalence [1]. Prior to the 1980s, general practitioners in the Netherlands were reluctant to label asthmatic symptoms in children as having a diagnosis of asthma, since the social consequences and the impact of this diagnosis were far-reaching. Together with the steroid phobia in the general public at that time, this probably resulted in an underdiagnosis of asthma. This changed considerably due to the introduction and subsequent widespread use of inhaled corticosteroids in the following years. It is interesting to note that when we compared two identical surveys in Germany and the Netherlands from 1995 and 1997, in Dutch-German borderland, the asthma diagnosis was more prevalent in Dutch children with recent asthmatic complaints (50-60\%), whereas $>90 \%$ of the German children with recent asthmatic 